\title{
Effects of Osteopontin in Preadipocyte Differentiation
}

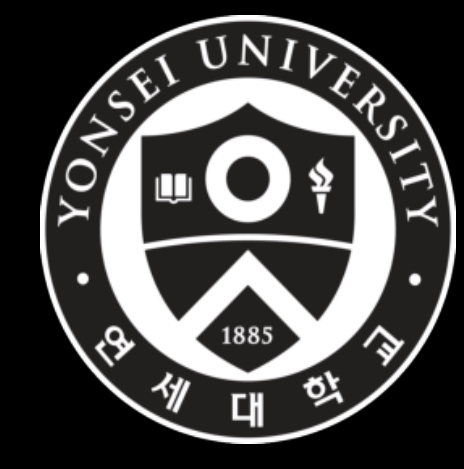

\section{Introduction}

Obesity is caused by increased adipose tissue mass with increased size and numbers of adipocytes. This can lead to many complications including diabetesassociated insulin resistance [1].

Browning of the white adipose tissue is beneficial in the prevention of obesity and metabolic diseases [2].

Adipocyte regulations are affected by osteopontin (OPN) which is a cytokine secreted primarily from bones [3].

OPN mediates various biological functions such as cell survival and plays a role in inflammation [4]. However, effect of OPN on adipogenesis is unclear.

\section{Specific Aims}

In this study, we investigated the effects of osteopontin on adipogenesis using 3T3-L1 preadipocytes (Fig. 1).

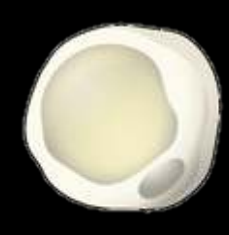

White adipocyte

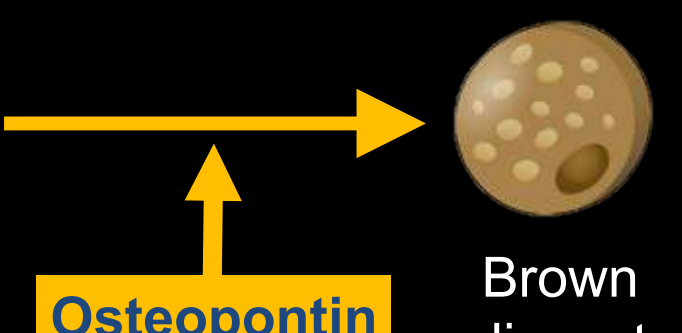

dipocyte

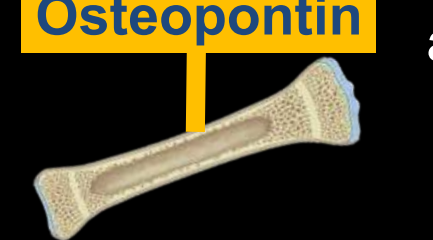

Fig. 1. Effect of osteopontin on adipogenesis.

\section{Methods}

Table. 1. Experimental Group.

\begin{tabular}{|c|c|c|c|c|}
\hline Time & \multicolumn{4}{|c|}{ OPN Dose (nM) } \\
\hline 2 hour & 0 & 1 & 10 & 100 \\
\hline 2 day & 0 & 1 & 10 & 100 \\
\hline
\end{tabular}

Preadipocytes were stimulated with 0,1 , 10 and 100 nM OPN for either 2 hours or 2 days (Table. 1 ).

Proteins were assessed by Western blot analysis. Immunoreactive proteins were visualized by exposure to X-ray film. Protein bands were shown by image scanning. GAPDH was used as a control.

\author{
Jeongkun Lee and Chi Hyun Kim \\ Department of Biomedical Engineering \\ Yonsei University \\ Republic of Korea
}

\section{Results}

OPN treatment increased UCP-1, and PRDM16 expressions compared to the control (Fig. 2, 3).

OPN treatment significantly decreased the size of lipid droplets, especially in the 2 hour treatment group (Fig. 4).

\begin{tabular}{|c|c|c|c|c|}
\hline OPN (UM) & 0 & 1 & 10 & 100 \\
\hline UCP-1 & * & $=$ & & $=$ \\
\hline PRDM16 & $n=$ & newes & ensess & 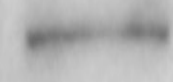 \\
\hline p-ERK & 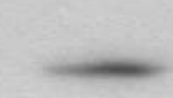 & - & $=$ & - \\
\hline C/EBP $\beta$ & -1 & - & $=$ & $=$ \\
\hline PPARY & $m$ & 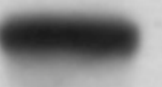 & 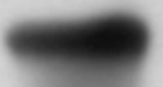 & $=$ \\
\hline GAPDH & & & 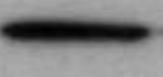 & - \\
\hline
\end{tabular}

Fig. 2. Expression of proteins with various concentrations of OPN in 3T3-L1 preadipocytes ( 2 hours).

\begin{tabular}{|c|cccc|}
\hline OPN (uM) & 0 & 1 & 10 & 100 \\
\hline UCP-1 & & & & \\
\hline PRDM16 & & & \\
\hline p-ERK & & & & \\
\hline C/EBP $\beta$ & & & & \\
\hline PPARY & & & & \\
\hline GAPDH & & & \\
\hline
\end{tabular}

Fig. 3. Expression of proteins with various concentrations of OPN in 3T3-L1 preadipocytes (2 days).

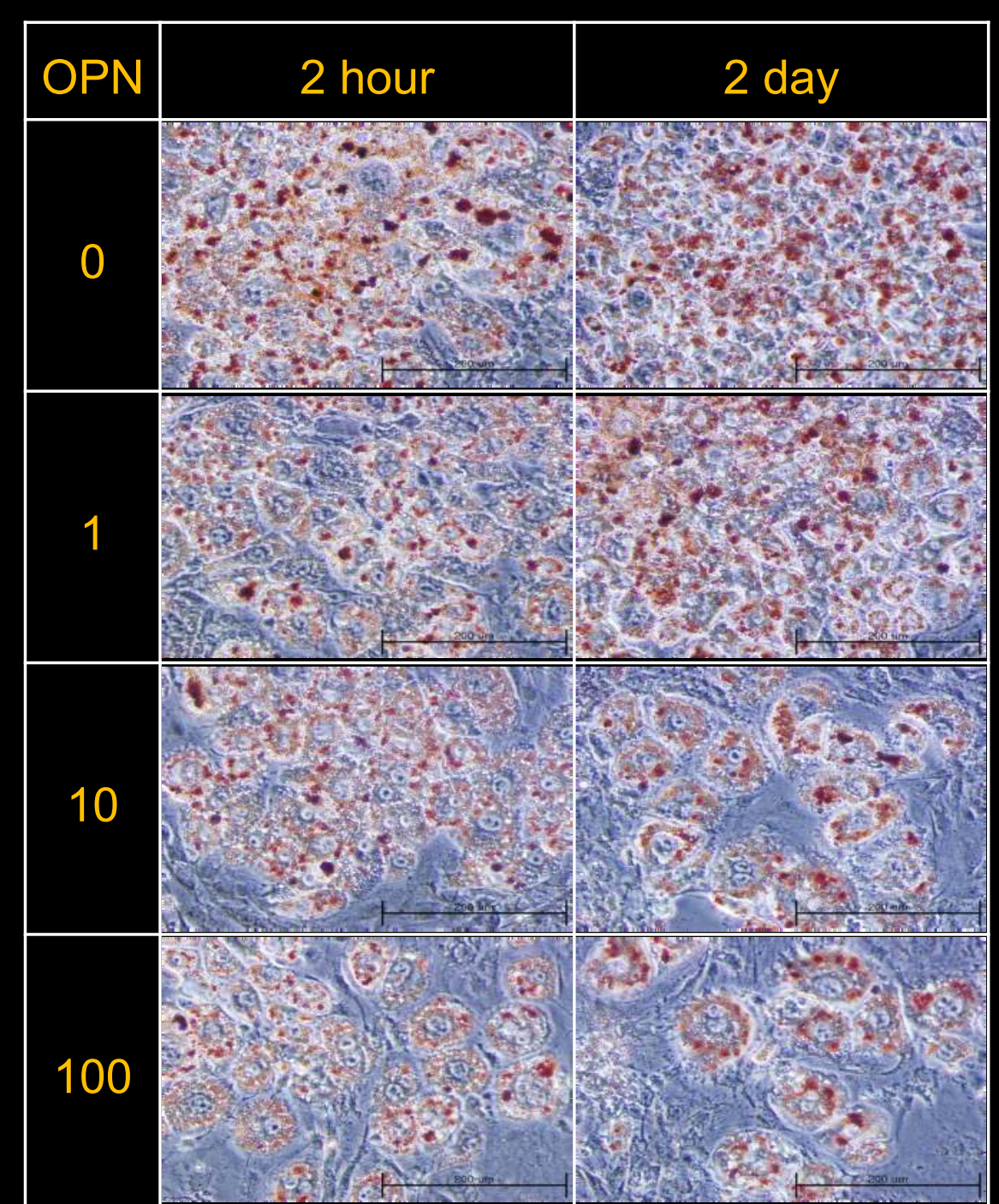

Fig. 4. After OPN treatment in each group, Oil Red O staining was conducted to detect the lipid droplets in 3T3-L1 preadipocytes.

\section{Discussion}

OPN induced activation of brown adipogenesis marker proteins from white adipocytes.

OPN may promote the maturation of preadipocytes into brown adipocytes (Fig. 5).

These results suggest that physical exercise may help treat obesity by turning white adipocytes into brown adipocytes by OPN.

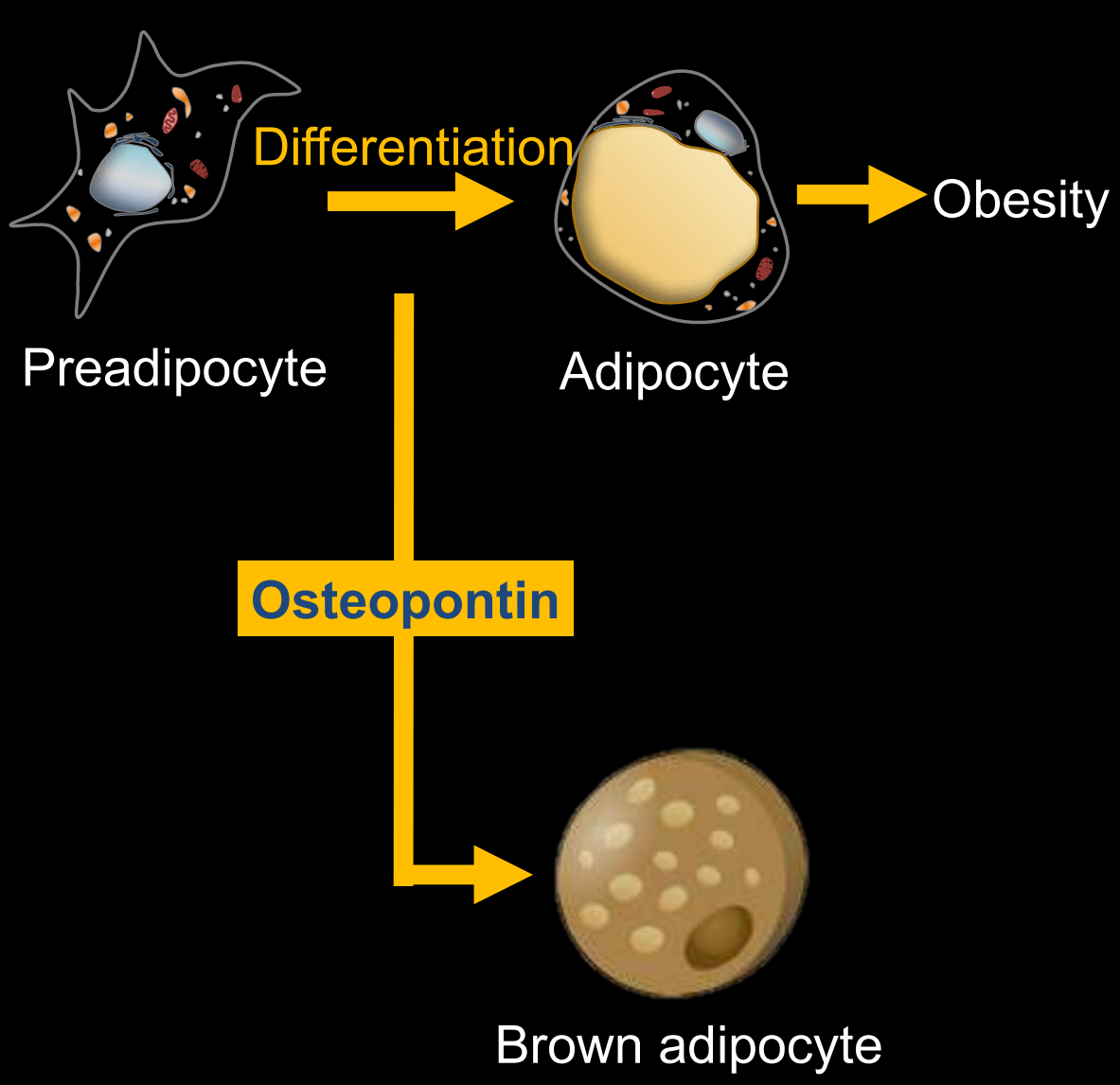

Fig. 5. Regulation of adipogenesis by osteopontin in 3T3-L1 preadipocytes.

\section{References}

Kahn et al., Mechanisms linking obesity to insulin resistance and type 2 diabetes, Nature, 2006.

Whittle et al., Using brown adipose tissue to treat obesity - the central issue, Trends Mol Med, 2011.

Zhong et al., Osteopontin-induced brown adipogenesis from white preadipocytes through a PI3K-AKT dependent signaling, Biochem Biophys Res Commum, 2015.

Lund et al., The role of osteopontin in inflammatory processes, J Cell Commun Signal, 2009.

\section{Acknowledgement}

This research was supported by Basic Science Research Program through the National Research Foundation of Korea (NRF) funded by the Ministry of Education. 